\title{
Evaluating networked drug checking services in Toronto, Ontario: study protocol and rationale
}

\author{
N. Maghsoudi ${ }^{1}, 2$, K. McDonald ${ }^{1}$, C. Stefan ${ }^{3}$, D. R. Beriault ${ }^{4,5}$, K. Mason $^{6}$, L. Barnaby ${ }^{7}$, J. Altenberg ${ }^{6}$, R. D. MacDonald ${ }^{8,9}$, \\ J. Caldwell ${ }^{10}$, R. Nisenbaum ${ }^{11,12}$, P. Leece ${ }^{12,13,14}$, T. M. Watson ${ }^{15}$, K. W. Tupper ${ }^{16}$, L. Kufner ${ }^{7}$, A. I. Scheim ${ }^{1,17}$, \\ D. Werb ${ }^{1,2,17^{*}}$ (D) and Drug Checking Services in Toronto Working Group
}

\begin{abstract}
Background: The increasing incidence of fatal opioid overdose is a public health crisis in Canada. Given growing consensus that this crisis is related to the presence of highly potent opioid adulterants (e.g., fentanyl) in the unregulated drug supply, drug checking services (DCS) have emerged as part of a comprehensive approach to overdose prevention. In Canada's largest city, Toronto, a network of DCS launched in 2019 to prevent overdose and overdose-related risk behaviors. This network employs mass spectrometry technologies, with intake sites co-located with supervised consumption services (SCS) at three frontline harm reduction agencies. The protocol and rationale for assessing the impact of this multi-site DCS network in Toronto is described herein. The aims of this study are to (1) evaluate the impact of DCS access on changes in and factors influencing overdose and related risk behaviors, (2) investigate the perceived capacity of DCS to prevent overdose, and (3) identify composition (qualitative and quantitative) trends in Toronto's unregulated drug supply.
\end{abstract}

Methods: We will use a parallel-mixed-methods design with complementary data sources (including data from chemical analysis of drug samples, quantitative intake and post-test surveys, SCS, coroners, paramedic services, and qualitative interviews), followed by a meta-inference process wherein results from analyses are synthesized.

Results: Whereas most DCS globally target "recreational drug users," in Toronto, this networked DCS will primarily target marginalized people who use drugs accessing frontline services, many of whom use drugs regularly and by injection. This evolution in the application of DCS poses important questions that have not yet been explored, including optimal service delivery models and technologies, as well as unique barriers for this population. Increasing information on the unregulated drug supply may modify the risk environment for this population of people who use drugs.

Conclusions: This study addresses evidence gaps on the emerging continuum of overdose prevention responses and will generate critical evidence on a novel approach to reducing the ongoing high incidence of drug-related morbidity and mortality in Canada and elsewhere.

Keywords: Drug checking services, Harm reduction, Overdose, Drug market monitoring

\footnotetext{
* Correspondence: dwerb@ucsd.ed

${ }^{1}$ Centre on Drug Policy Evaluation, Li Ka Shing Knowledge Institute, St. Michael's Hospital, 30 Bond Street Toronto, Ontario M5B 1 W8, Canada ${ }^{2}$ Institute of Health Policy, Management and Evaluation, University of Toronto, Toronto, Ontario, Canada

Full list of author information is available at the end of the article
} to the Creative Commons license, and indicate if changes were made. The Creative Commons Public Domain Dedication waiver (http://creativecommons.org/publicdomain/zero/1.0/) applies to the data made available in this article, unless otherwise stated. 


\section{Background}

The increasing incidence of fatal opioid overdose is a public health crisis in Canada. From January to September 2018, there were 3286 opioid-related deaths in Canada, corresponding to a death rate of 11.8 per 100,000 population (compared to 11.1 and 8.4 per 100,000 population in 2017 and 2016, respectively) [1]. In the western province of British Columbia, opioid overdose deaths have contributed to a decline in life expectancy, particularly among the most socioeconomically disadvantaged and marginalized communities [2]. In the province of Ontario, opioid overdose is now the leading cause of accidental death for young people, with one of every eight deaths among individuals aged 25 to 34 years involving an opioid [3]. With 308 opioid-related deaths in 2017, the country's largest city, Toronto, Ontario, has experienced a $125 \%$ increase in deaths compared to 2015 [4]. There is now a consensus that the rising incidence of overdose is related in large part to the emergence of highly potent opioids, such as fentanyl and fentanyl analogs, in the unregulated drug supply. For example, fentanyl is present in an increasing number of overdose fatalities in Canada, though overdose deaths excluding fentanyl have remained relatively stable [5]. In Ontario, the presence of fentanyl in opioid-related deaths increased by $548 \%$ between 2006 and 2015 [6]. In 2017, there were 1265 opioid-related deaths in the province and fentanyl was present in $63.6 \%$ of these deaths; in contrast, fentanyl was present at death in $40.7 \%$ of 867 opioidrelated deaths in 2016 [7]. This trend has thus accelerated in recent years, and fentanyl has overtaken heroin as the opioid most commonly present in opioid overdose deaths in Toronto [4]. In response, Canada's federal government has characterized the current situation as a national public health crisis and allocated CAD\$116 million in 2017 to a public health emergency response coordinated by Health Canada $[8,9]$, while the Government of Ontario committed CAD $\$ 280$ million over 3 years to address the opioid overdose crisis [10].

Against this backdrop [11], drug checking services (DCS) have emerged as part of a comprehensive approach to preventing overdose mortality $[12,13]$. Stakeholders-including the Federal Standing Committee on Health [14], Toronto Board of Health [15], and Mayors' Task Force on the Opioid Crisis [16]-have identified DCS as a critical tool within a comprehensive plan to prevent overdoses related to the use of high-potency unregulated opioids. As a harm reduction intervention available in Europe since the 1990s, DCS provide information on the composition of drugs to their clients in order to facilitate more informed drug-related decision-making and to increase the capacity of individuals to avoid ingesting unanticipated toxic substances, which can lead to overdose and death $[17,18]$. By aggregating analytic data on drug samples, DCS can also provide insight into trends in the unregulated drug supply [19-26]. The Canadian federal government has identified DCS as a key component of their response to the opioid overdose crisis and has committed to authorizing and funding pilot projects providing DCS at supervised consumption services (SCS) [27]. This includes funding over 5 years for the implementation of a multi-site DCS network in Toronto with the primary aim of reducing overdose deaths among structurally vulnerable individuals reliant on the unregulated opioid supply [28]. Launched in 2019, the DCS network in Toronto is located within frontline harm reduction agencies offering SCS and employs liquid chromatography and gas chromatography-mass spectrometry (LC- and GC-MS), the gold standards in forensic drug analysis [29], making Toronto's DCS unique among Canadian DCS [30].

To date, evaluations of DCS have been limited to "recreational drug users" in nightlife settings in Europe [19, 31, 32], and no DCS employing LC- or GC-MS analysis for point of care services and operating within SCS have been scientifically evaluated. Herein, we describe the methodology for a study assessing the impact of a multi-site DCS network in Toronto. The overall goal of the this study is to determine whether access to DCS, to be co-located with SCS at three frontline harm reduction agencies in Toronto, is associated with a reduction in the incidence of overdose and related risk behaviors resulting from the consumption of unknown or toxic unregulated drugs by people who use drugs accessing frontline services, including young people who use drugs. The study has three specific aims: (1) evaluate the impact of DCS access on changes in and factors influencing overdose and related risk behaviors, (2) investigate the perceived capacity of DCS to prevent overdose, and (3) identify composition (qualitative and quantitative) trends in Toronto's unregulated drug supply. Given the limited evidence on the influence and impact of DCS for subpopulations of people who use drugs regularly or by injection, this research study will represent a novel contribution to the scientific literature and provide important insights on DCS as an overdose prevention intervention. The protocol and rationale for this study is itself an important contribution to the literature, as it provides full study transparency and will be useful to researchers planning to evaluate DCS in other jurisdictions.

\section{Methods}

\section{Conceptual framework}

We employed Rhodes' risk environment framework, which posits that physical, social, economic, and policy environments influence an individual's health decisionmaking [33]. Drug policy changes in Canada-such as expanded access to naloxone (a medication that blocks the effects of opioid overdose), the adoption of legislation providing amnesty from drug possession charges for 
those calling emergency services in an overdose situation, and the scale-up of SCS-have modified the risk environment for people who use drugs to allow this population to avoid a range of drug-related risks. However, these responses are generally limited to addressing the needs of individuals during or after drug use, rather than before. In contrast, DCS are intended to provide people who use drugs with critical information so they can make informed decisions and take action prior to their use of unregulated drugs, thereby possibly preventing overdose from occurring in the first place. This is important given that an examination of the current risk environment experienced by people who use drugs in Toronto suggests that, while the emergence of fentanyl and fentanyl analogs in the unregulated drug supply is a macro-level factor in the increased incidence of fatal overdose and drug-related harm, social (e.g., experiences of trauma, ongoing stigma), economic (e.g., poverty), and policy (e.g., criminalization of drugs, lack of information on drug supply, low levels of access to social services) barriers constrain the capacity of individuals to avoid overdose risk [34, 35]. Applying Rhodes' risk environment framework to this research study, DCS are defined as meso-level interventions that have the capacity to expand the range of choices that individuals reliant on the unregulated drug supply have to avoid overdose. This conceptual framework will guide variable selection for quantitative analyses and domains of interest for qualitative interviews. For instance, the study will examine the neighborhood in which drugs were purchased given that this factor shapes the meso- and micro-level economic risk environments [36] experienced by participants that may influence overdose risk. Gender and age will also be considered in the study to situate experiences of access to DCS and resulting outcomes given that the risk environment for drug-related harms is different across genders and ages [37, 38]. Qualitative interviews will investigate the influence of DCS on individuals' perceived barriers to adopting overdose risk reducing behaviors.

\section{Approach}

By employing complementary data sources-including data from analysis of drug samples, quantitative intake and post-test surveys, SCS, coroners, paramedic services, and qualitative interviews - this study will comprehensively evaluate the impact of DCS on a range of policyrelevant drug-related outcomes, while considering the impacts of co-located SCS (refer to Table 1: Measures, Outcomes, and Data Sources). A mixed-methods approach followed by a meta-inference process will be used to maximize multiple data sources and synthesize results of quantitative and qualitative analyses.

\section{Study partners}

Three frontline harm reduction agency partners-Parkdale Queen West Community Health Centre (Queen West site), South Riverdale Community Health Centre, and The Works at Toronto Public Health-have been funded as DCS intake sites. With the exception of The Works, which is a public health agency, the remaining two partnering frontline agencies are community health centers, and all agencies combine harm reduction service delivery with access to other services, including primary care, mental health care, and social service programs. As DCS intake sites, agencies will collect drug samples from

Table 1 Measures, outcomes, and data sources

\begin{tabular}{|c|c|}
\hline Measures and outcomes & Data sources \\
\hline \multicolumn{2}{|c|}{ Aim 1: Evaluate the impact of DCS access on changes in and factors influencing overdose and related risk behaviors } \\
\hline Self-reported overdose among those that access DCS and those that do not & $\begin{array}{l}\text { - Quantitative survey data (intake) } \\
\text { - SCS client data }\end{array}$ \\
\hline $\begin{array}{l}\text { Proportion of participants reporting increase in protective behaviors (not using alone, carrying } \\
\text { naloxone, use of SCS, consultation with staff, smaller/) "tester" dosage, discarding toxic substances) }\end{array}$ & $\begin{array}{l}\text { - Quantitative survey data (intake/post-test) } \\
\text { - SCS client data }\end{array}$ \\
\hline Proportion of participants reporting they gained, intend to use, and/or used knowledge and skills & $\begin{array}{l}\text { - Quantitative survey data (post-test) } \\
\text { - Qualitative interviews }\end{array}$ \\
\hline \multicolumn{2}{|l|}{ Aim 2: Investigate the perceived capacity of DCS to prevent overdose } \\
\hline Characterize participant perceptions on the capacity of DCS to alter the risk of overdose & - Qualitative interviews \\
\hline Identify participant perceptions of contexts, facilitators, and barriers to the use of DCS & - Qualitative interviews \\
\hline \multicolumn{2}{|l|}{ Aim 3: Identify trends in the composition (qualitative and quantitative) of the unregulated drug supply in Toronto } \\
\hline Number of analysis results detecting composition different from participant expectations & $\begin{array}{l}\text { - Quantitative survey data (intake) } \\
\text { - Drug sample analysis data }\end{array}$ \\
\hline Increase in accuracy and timeliness of alerts in response to dangerous drug trends & $\begin{array}{l}\text { - Drug sample analysis data } \\
\text { - Qualitative interviews }\end{array}$ \\
\hline $\begin{array}{l}\text { Spatial association between frontline harm reduction agencies with DCS and fatal overdose, } \\
\text { and changes over time }\end{array}$ & $\begin{array}{l}\text { - First responder and coroner data } \\
\text { (from OCC and TPaS) }\end{array}$ \\
\hline
\end{tabular}


participants, administer intake and post-test surveys, facilitate transport of samples to clinical laboratories, and communicate analysis results to participants along with relevant harm reduction information. Notably, Parkdale Queen West Community Health Centre (Queen West site) also houses TRIP Project, a program providing information and resources on harm reduction and safer sex to youth in nightlife and party settings.

Clinical laboratory partners at the Centre for Addiction and Mental Health and St. Michael's Hospital-two urban hospitals with specialized programs for substance use-will analyze samples of drugs from participants at frontline harm reduction agencies using LC- and GCMS techniques and guide the aggregation of analysis data across sites to identify composition (qualitative and quantitative) trends in the unregulated drug supply.

To bolster the relevance of findings and ensure effective knowledge translation strategies that address the needs of key populations, a Community Advisory Board made up of people who use drugs will advise on all aspects of the design, analysis, interpretation, and knowledge translation activities of this study [39]. Additionally, protocols for research methods and outcome measures will be aligned as much as possible with DCS implemented in other Canadian jurisdictions, including British Columbia and the capital city of Ottawa, Ontario, to maximize the potential comparability of findings across distinct settings.

\section{Data collection}

\section{Intake and post-test surveys (Aim 1)}

Intake and post-test surveys will be administered to all consenting DCS clients at the three sites in Toronto, including participants who access DCS once and those who access DCS multiple times, allowing for a combined use of crosssectional and longitudinal approaches. Surveys will be administered by frontline staff on an electronic tablet with the option for self-administration depending on the needs of the participant. Guided by the abovementioned conceptual framework, demographic and drug-using factors will be collected at first visit to DCS via a baseline intake survey to assess the influence of additional vulnerabilities facing key populations. An anonymous identifier will be created in the baseline intake survey and linked to intake surveys completed at subsequent visits. Questions on the intake survey, administered at every visit to DCS, include the following: What substance participants believe they are having tested?; When and where they purchased the substance?; and, If they are sharing the results with others, with how many people? Post-test surveys conducted immediately after analysis results are provided will query participants about their intended behaviors in response to analysis results, as well as allow frontline staff to record other services accessed (e.g., SCS, naloxone) and observed behaviors (e.g., discarding remainder of drug that was tested).

\section{Sample size (Aim 1)}

In lieu of randomization, which is neither feasible nor ethical given the type of service to be examined in the present study, a community recruitment model will be employed whereby a survey of all consenting DCS clients is undertaken (i.e., the sampling frame consists of all clients of DCS). Since study participants are recruited "passively" through their use of the service, we are unable to estimate the proportion of surveys to be obtained from unique versus repeat clients until after DCS are implemented. We therefore cannot determine the total person-time data that will be collected. We note, however, that $75 \%$ of feasibility survey respondents recruited from frontline agencies reported being willing to access DCS. The total population of structurally vulnerable people who use drugs in Toronto is estimated at 9000, among whom $20 \%$ are willing to use SCS [35]; it is estimated that $n=1800$ unique individuals who use drugs access frontline agencies. Previous literature suggests social desirability bias in reports of willingness to access harm reduction services [40]; we therefore estimate an approximate total of $n=1080$ DCS clients.

\section{SCS client survey data (Aim 1 substudy)}

An ongoing longitudinal cohort is collecting data from people who inject drugs who do and do not use SCS at the three frontline agencies participating in the DCS network. This allows for harmonization of survey tools across both projects. As such, for participants consenting to linkages, data from a standardized SCS cohort survey (e.g., age, gender, neighborhood of residence, and drug-using factors) and data from the SCS pre/post injection assessment form (e.g., overdose outcomes, whether emergency medical attention was provided [i.e., SCS staff response and/or call to 911 for paramedic response], naloxone administered) will be accessible for consenting participants. This will also allow for a substudy consisting of a comparison of outcomes (i.e., overdose incidence and related risk behaviors) among frontline agency clients who access SCS exclusively, who access both SCS and DCS, and who access neither.

\section{Qualitative interviews (Aim 2)}

In-depth interviewing will investigate participant perceptions of the contexts, facilitators, and barriers to the use of DCS and its capacity to influence overdose risk. We will also specifically investigate participant perspectives on the influence of gender on both DCS access and overdose risk, given the lack of data on this topic and the known contribution of gender to drug-related harms (i.e., women are less likely to manage illegal drug acquisition and inject themselves compared with men) [41-44]. We will employ purposive sampling to achieve gender diversity (including transgender individuals) and to ensure youth representation 
drawn from Toronto's structurally vulnerable drug-using population [35]. Two interview waves will be conducted, and both will include 10 interviews with participants from each of the three SCS/DCS sites, which includes 5 interviews with youth accessing DCS via the TRIP Project (total $n$ per wave $=30$; total $n=60$ ). Two waves of interviews will also be held with institutional stakeholders providing DCS services $(n=10$ per wave; $n=20)$ to provide insight into the capacity and limits of DCS to prevent overdose and related risk behaviors.

\section{Analysis of drug samples (Aim 3)}

Mass spectrometry (MS) is a versatile technology that in combination with liquid (LC)- or gas (GC)-chromatographic techniques can identify virtually any chemical compound [45]. There are various MS instrument configurations, but essentially MS allows for highly accurate identification of a compound by measuring its mass to charge ratio $(\mathrm{m} / \mathrm{z})$ and analyzing its fragmentation pattern (mass spectrum) [29]. Separation of compounds is accomplished through the use of LC- or GC-columns. Importantly, LC- and GC-MS methods can distinguish between structural and/or functional analogs and can therefore identify the presence of highly potent toxic opioids such as fentanyl and its analogs within samples that contain multiple opioids [3, 46-48]. Drug samples will be transported to nearby clinical laboratories for analysis with LC- and GC-MS. Analysis results on composition (qualitative and quantitative) of drug samples will be communicated within one to two business days to frontline staff, although the ultimate goal of the pilot project is to reduce turnaround time to $<2 \mathrm{~h}$; this evaluation will seek to identify process refinements that may expedite faster analysis and dissemination of results. Frontline staff will communicate results along with tailored harm reduction strategies to clients either in person or by phone.

\section{Coroner and paramedic services (Aim 3)}

Data-sharing agreements with the Office of the Chief Coroner for Ontario (OCC) and Toronto Paramedic Services (TPaS) will cover both de-identified individual- and aggregated-population level data on the incidence of fatal and non-fatal overdose events, including toxicology, temporal information, and geographic locations.

\section{Estimated overdose incidence (Aim 3)}

While no formal estimates of the overdose rate among people who use drugs in Toronto have been derived, 1002 overdose calls (fatal and non-fatal combined) were made to Toronto Paramedic Services between January 2018 and December 2018 in neighborhoods adjacent to the three frontline DCS sites, representing $31 \%$ of all calls across Toronto [49]; these neighborhoods are also those with the greatest density of people who use drugs [35]. We also note that the incidence of $>1000$ overdose events recorded near DCS sites implies a relatively high event rate among potential DCS clients.

\section{Analytic plan Multivariable model of participant overdose risk behaviors (Aim 1)}

For participant survey binary outcomes, we will employ either population-averaged models (i.e., generalized estimating equations; GEE) or subject-specific models (generalized linear mixed models; GLMM) with binomial distribution and logit link. The primary outcome is recent (i.e., past 6 months) self-reported overdose; the primary exposure is a categorical measure of frequency of DCS access over the past 6 months. GEE will be used if participant visits are equally distributed over time and if we have low levels of missing data. In the case of unequal distribution of participant visits over time and/or substantial missing data, we will employ GLMM. These models efficiently address problems of missing data and variable timing of subject visits. Both GEE and GLMM are suited to the analysis of longitudinal data, as they can account for both within-subject correlation and between-subject differences with results also remaining highly interpretable. We will initially model temporal correlations between subject responses using an autoregressive structure, with subject-specific variances and correlations assumed to decline exponentially over time; this is critical given that we anticipate variance in client follow-up times. GLMM models will also include independent time-varying variables from the linked intake and post-test surveys as potential confounders. This will allow for a "pre versus post" test evaluation using longitudinal DCS data. To assess potential bias as a result of loss to follow-up, sociodemographic and drug-related factors collected on the intake and post-test surveys will be compared among participants who only completed a baseline DCS visit and those that accessed the service repeatedly. Multilevel multiple imputations may also be considered if needed.

\section{Thematic analysis (Aim 2)}

Thematic analysis will be employed to guide qualitative data collection and simultaneous, iterative analysis to identify themes within the data [50]. Transcripts will be reviewed multiple times by co-authors to draw on key codes, and analysis will be completed once themes reach saturation [51]. Analysis will also disaggregate responses by age, gender, racial group, sexual orientation, and drug-using behavior to identify similarities and conflicting perspectives. Respondent verification, constant comparative analysis, and multiple coding will be employed in the analysis $[52,53]$. 
Longitudinal analysis of time-updated overdose data (Aim 3) Using OCC and TPaS data, the influence of DCS on the annual incidence of fatal and non-fatal overdose events will be characterized, while adjusting for underlying trends over time. Following others [54], conditional fixed effects Poisson models will be employed to test overdose incidence counts in geographic units that do and do not have DCS present, while adjusting for the presence of SCS. This approach allows for assessment of the estimated effect of DCS by analyzing the fluctuation in incidence rates in areas before and after DCS implementation, and by then comparing the relative change in incidence to other geographic areas. Disentangling the independent effects of DCS from those of SCS presents analytic challenges. However, SCS in Toronto were operational before DCS. This stepwise implementation represents an ideal natural experiment to assess the independent effect of DCS.

\section{Spatial patterns of fatal and non-fatal overdose in Toronto (Aim 3)}

Employing approaches previously used to assess the association between risky drug-related behaviors and frontline services [55], data from the OCC and TPaS will be used to assess the spatial association between frontline harm reduction agencies with DCS and overdose events. This will be done by comparing geographic units that have DCS and SCS co-located with sites that only have SCS located, compared with sites that have neither. Changes over time in the incidence of overdose in these units will be determined by employing geographic information systems approaches. First, spatial buffers around each DCS site will be generated, and TPaS and OCC data will be used to capture the spatial location of nonfatal and fatal overdoses in surrounding areas. Significance testing will then be done by analyzing dissemination blocks (the smallest geographic unit defined by Statistics Canada with a population of 400-700 people). Toronto experienced 1542 non-fatal and 111 fatal overdoses over a recent 6-month period (August 2017February 2018), and based on existing spatial data, it is estimated that the range of annual overdose events per dissemination block will range from 0 to $>100$. This exploratory approach represents a simple descriptive test to determine the spatial correlation of overdoses and DCS. Second, to further account for potential confounding of the association between DCS and spatial patterns of overdose as a result of co-location of SCS and DCS [56], the mean distances between fatal overdoses (OCC data) in neighborhoods in which SCS are implemented but have not yet implemented DCS will be compared. Third, geographically weighted regression (GWR) will be employed to determine the spatial heterogeneity between the location of overdoses (dependent variable) and location of DCS (independent variable), while accounting for the presence of SCS as above. GWR calculates local spatial statistics by fitting a weighted regression equation for each dissemination block in the study area to assess local variation [57]. Local statistics will then be compared with the global statistic for the entire geographic area (i.e., the Province of Ontario), and blocks exhibiting local variation differing from the global statistic will be identified (including by undertaking a sub-analysis restricted to blocks within the City of Toronto). Using GWR, it will be determined whether those blocks exhibiting local variation in overdose incidence are more or less likely to also contain DCS. Fourth, the local annual incidence rate of overdose for each block will be determined. The Wilcoxon signed rank-test has previously been shown to be effective at assessing fluctuations in the annual rate of fatal and non-fatal overdose [56], and will allow a determination of slope differences in the overdose rate in blocks that do and do not have DCS.

\section{Mixed-methods analysis}

A parallel mixed-methods design followed by a metainference process will be employed [58]. Specifically, qualitative research findings will be used to interpret survey- and laboratory-based data collection within the context of the lived experience of participants. This approach has been shown to be effective in generating an understanding of the social dimensions underpinning quantitative outcomes (e.g., use of and outcomes related to DCS, or the incidence and spatial patterns of overdose) [59-61]. Qualitative data collection and interpretation will also be used to refine the quantitative data collection process to better capture relevant factors.

\section{Ethics, consent, and permissions}

Research Ethics Board approval has been obtained for this research study from Providence St. Joseph's and St. Michael's Healthcare, Toronto Public Health, Centre for Addiction and Mental Health, and University of Toronto. Client provision of informed consent is integrated into the baseline intake survey, although clients that are unwilling or unable to provide informed consent will still be provided access to DCS. Data will be securely stored at St. Michael's Hospital.

\section{Results}

The study addresses evidence gaps on the emerging continuum of responses to the opioid overdose crisis in Canada and elsewhere. Internationally, DCS have only targeted "recreational drug users," and no DCS colocated with SCS and using LC- or GC-MS have been scientifically evaluated. The study therefore builds upon new and incremental lines of inquiry by evaluating the potential impact of DCS within a suite of harm 
reduction interventions tailored for populations at highest risk of overdose [62]. Given the rise in opioid overdose events and fatalities in Canada, the USA [63], and the UK [64], findings will provide decision makers with critical data on the potential impact of an innovative DCS model on overdose risk.

DCS in Toronto have been integrated within public health and community health agencies with existing SCS, thereby combining harm reduction service delivery with access to other services including primary care, mental health care, social service programs, and scientific evaluation. The implementation of DCS will allow frontline harm reduction agencies to extend their services by providing clients with timely information on the composition (qualitative and quantitative) of their drugs. While DCS are not a panacea for drug-related harms, increasing information on the unregulated drug supply may disrupt the risk environment for people who use drugs by equipping them with tailored and highly relevant information related to their substance use, as well as by increasing transparency and accountability in the drug market by reducing information asymmetries between buyers and sellers throughout the supply chain. Evidence from European DCS indicates that DCS benefits can extend beyond those individuals that engage directly with the service, as analysis results are often communicated to others, thus multiplying the preventive effect for a broader population of people who use drugs [65]. Although DCS can play an important role in drug market monitoring and therefore have the potential to influence drug market activities, the absence of legally regulated markets for illegal drugs creates a paradox wherein people who use drugs may become aware of dangerous adulterants in their drugs but have no viable option for the replacement of the analyzed substance. Particularly for those facing intersecting vulnerabilities such as poverty and substance-use disorders, increased information on substance composition may be insufficient to modify their risk environment. In cases where information from DCS does not effectively reduce overdose risk for clients, other policy approaches and interventions-such as scaling up low-barrier access to managed opioid programs-should be considered. Importantly, DCS have the potential to attract clients who may otherwise not access drug-related services-as has been demonstrated by DCS in Europe [18, 22, 66]-and given the co-location of DCS with other services, DCS may support increased uptake of other harm reduction, primary care, mental health care, and social services among people who use drugs. While such an integrated model of harm reduction services offers the promise of a "one-stop shop" with the potential to improve health outcomes for people who use drugs, drawbacks such as reduced anonymity may pose barriers for some clients.
Accompanying evaluations of DCS and SCS will examine different models of service provision, under what conditions benefits can be maximized, suitability for specific subpopulations, and a variety of indicators including successful referrals to other services.

\section{Discussion}

A crucial difference between the adoption of DCS in Toronto and the longstanding use of this harm reduction service throughout Europe is the targeted subpopulation of people who use drugs. Whereas most DCS globally target "recreational drug users," in Toronto, DCS will primarily target marginalized people who use drugs accessing frontline services, many of whom use drugs regularly and by injection. This evolution in the application of DCS poses important questions that have not yet been explored, including optimal service delivery models and technologies, as well as unique barriers for this population. As the only DCS in Canada employing LCand GC-MS analysis for point of care services, it will be important to compare results with DCS currently operating in British Columbia and Ottawa employing different and less sophisticated technologies (e.g., Fourier-transform infrared spectroscopy, fentanyl test strips), including in terms of the capability of technologies to detect drug market trends and respond to local drug use patterns. Although employing LC- and GC-MS for the analysis of drug samples has the potential to offer clients more information than the use of other technologies, the time required to receive analysis results may not be suitable for all populations of people who use drugs. It remains to be seen whether participants will consume their substance before receiving analysis results, and if they will modify their behaviors in response to analysis results, particularly when a highpotency opioid is detected.

\section{Limitations}

The study has limitations typical of community-based intervention evaluations. Since denying access to DCS is not ethical or feasible, the recruitment process is being integrated directly into the delivery of DCS. While this will allow for the collection of data from a large proportion of the population of DCS clients, it does present limitations. For instance, it is not possible to estimate the number of individuals that will access DCS and provide data, nor the total person-time of data. Second, we note challenges in disentangling the impact of SCS and DCS on overdose incidence and related behaviors given that these two interventions will be co-located in our study setting. However, the 1-year delay between the implementation of SCS and that of DCS provides an ideal natural experiment to test the independent effects of these interventions. Further, the harmonization of data collection tools between SCS and DCS evaluations will 
further allow for the assessment of independent effects as we are able to evaluate outcomes among SCS clients who do and do not use DCS. Another limitation is the absence of a comparison group, except for the SCS subanalysis. A final challenge worth noting is that while we would prefer that participants create an anonymous identifier, since this would allow us to link their data from separate visits to DCS and therefore employ panel approaches, this will depend upon the willingness of clients to consent to participating in the research, creating an anonymous identifier, and consistently recording their survey responses under their identifier at subsequent visits.

\section{Conclusions}

Given that the impact of DCS has yet to be tested among people who use drugs at highest risk of overdose, this study will provide critical data for a range of stakeholders seeking to respond to opioid overdose crises in Canada and other settings impacted by unacceptably high rates of overdose mortality. Presenting the protocol and rationale for this study is important at this juncture, as transparency in methods will assist researchers in examining and developing DCS methods and evaluation, as well as enable the production of similar evaluations in other jurisdictions.

\section{Abbreviations}

DCS: Drug checking services; GC: Gas chromatography; GEE: Generalized estimating equations; GLMM: Generalized linear mixed models;

GWR: Geographically weighted regression; LC: Liquid chromatography; MS: Mass spectrometry; OCC: Office of the Chief Coroner for Ontario;

SCS: Supervised consumption services; TPaS: Toronto paramedic services

\section{Acknowledgements}

We are grateful for contributions of the Drug Checking Services in Toronto Community Advisory Board.

\section{Authors' contributions \\ DW and NM drafted the manuscript. All other co-authors reviewed and provided substantive input on the manuscript. All authors read and approved the final manuscript.}

\section{Funding}

This project is supported by Health Canada's Substance Use and Addictions Program and the St. Michael's Hospital Foundation.

Nazlee Maghsoudi is supported by a Canadian Institutes of Health Research (CIHR) Vanier Canada Graduate Scholarship. Ayden Scheim is supported by a CIHR Fellowship. Dan Werb is supported via a New Investigator Award from CIHR, an Early Researcher Award from the Ontario Ministry of Research, Innovation and Science, and an Avenir Award from the U.S. National Institute on Drug Abuse (NIDA DP2-DA040256).

\section{Availability of data and materials}

Not applicable.

\section{Ethics approval and consent to participate}

Research Ethics Board approval has been obtained for this research study from Providence St. Joseph's and St. Michael's Healthcare, Toronto Public Health, Centre for Addiction and Mental Health, and University of Toronto.

\section{Consent for publication}

Not applicable.

\section{Competing interests}

The authors declare that they have no competing interests.

\section{Author details}

${ }^{1}$ Centre on Drug Policy Evaluation, Li Ka Shing Knowledge Institute, St. Michael's Hospital, 30 Bond Street Toronto, Ontario M5B 1 W8, Canada. ${ }^{2}$ Institute of Health Policy, Management and Evaluation, University of Toronto, Toronto, Ontario, Canada. ${ }^{3}$ Clinical Laboratory and Diagnostic Services, Centre for Addiction and Mental Health, Toronto, Ontario, Canada. ${ }^{4}$ Department of Laboratory Medicine, St. Michael's Hospital, Toronto, Ontario, Canada. ${ }^{5}$ Department of Laboratory Medicine and Pathobiology, University of Toronto, Toronto, Ontario, Canada. ${ }^{6}$ South Riverdale Community Health Centre, Toronto, Ontario, Canada. ${ }^{7}$ Parkdale Queen West Community Health Centre, Toronto, Ontario, Canada. ${ }^{8}$ Toronto Paramedic Services, Toronto, Ontario, Canada. ${ }^{9}$ Faculty of Medicine, University of Toronto, Toronto, Ontario, Canada. ${ }^{10}$ Toronto Public Health, Toronto, Ontario, Canada. ${ }^{11}$ MAP Centre for Urban Health Solutions and Applied Health Research Centre, Li Ka Shing Knowledge Institute, St. Michael's Hospital, Toronto, Ontario, Canada.

${ }^{12}$ Dalla Lana School of Public Health, University of Toronto, Toronto, Ontario, Canada. ${ }^{13}$ Public Health Ontario, Toronto, Ontario, Canada. ${ }^{14}$ Department of Family and Community Medicine, University of Toronto, Toronto, Ontario, Canada. ${ }^{15}$ Institute for Mental Health Policy Research, Centre for Addiction and Mental Health, Toronto, Ontario, Canada. ${ }^{16} \mathrm{~S}$ chool of Population and Public Health, University of British Columbia, Vancouver, British Columbia, Canada. ${ }^{17}$ Division of Infectious Diseases and Global Public Health, University of California San Diego School of Medicine, La Jolla, CA, USA.

Received: 15 May 2019 Accepted: 6 November 2019

Published online: 28 January 2020

\section{References}

1. National Report: Apparent opioid-related deaths in Canada. Government of Canada; 2019

2. Ye X, Sutherland J, Henry B, Tyndall M, Kendall PRW. Impact of drug overdose-related deaths on life expectancy at birth in British Columbia. Health Promot Chronic Dis Prev Can. Research, Policy \& Practice. 2018;38(6).

3. Gomes T, Mamdani MM, Dhalla IA, Cornish S, Paterson JM, Juurlink DN. The burden of premature opioid-related mortality. Addiction. 2014;109(9):1482-8.

4. Toronto Overdose Information System Toronto. Toronto Public Health; 2018. Available from: https://www.toronto.ca/community-people/health-wellnesscare/health-inspections-monitoring/toronto-overdose-information-system/. Accessed 29 Apr 2019.

5. Illicit drug overdose deaths in BC: January 1, 2007 - June 30, 2017. B.C. Coroners Service; 2017. Retrieved from http://www2.gov.bc.ca/assets/gov/ public-safety-and-emergency-services/death-investigation/statistical/illicitdrug.pdf.

6. Gomes TGS, Martins D, et al. Latest trends in opioid-related deaths in Ontario: 1991 to 2015. Toronto: The Ontario Drug Policy Research Network; 2017.

7. Opioid-related morbidity and mortality in Ontario [Internet]. Public Health Ontario. 2017. Available from: https://www.publichealthontario.ca/en/ dataandanalytics/pages/opioid.aspx\#/trends. Accessed 29 Apr 2019.

8. Budget 2017 brings additional federal funding to fight the opioid crisis [press release]. Health Canada; 2017. Retrieved from https://www.canada.ca/ en/health-canada/news/2017/04/budget_2017_

bringsadditionalfederalfundingtofighttheopioidcrisis.html.

9. Federal Action on Opioids. Health Canada; 2017. Retrieved from https:// www.canada.ca/en/health-canada/services/substance-abuse/prescriptiondrug-abuse/opioids/federal-actions.html.

10. Ontario providing support to those affected by opioid crisis [press release]. Ontario Ministry of Health and Long-Term Care; 2017. Retrieved from https://news.ontario.ca/mohltc/en/2017/08/ontario-providing-support-tothose-affected-by-opioid-crisis.html.

11. Young MM, Pirie T, Buxton JA, Hosein FS. The rise of overdose deaths involving fentanyl and the value of early warning. Canadian Journal of Addiction. 2015;6:13.

12. Strike C, Watson TM. Losing the uphill battle? Emergent harm reduction interventions and barriers during the opioid overdose crisis in Canada. Int J Drug Policy. 2019.

13. Laing MK, Tupper KW, Fairbairn N. Drug checking as a potential strategic overdose response in the fentanyl era. Int J Drug Policy. 2018;62:59-66. 
14. Report and recommendations on the opioid crisis in Canada. 2016. Retrieved from http://publications.gc.ca/collections/collection_2016/parl/ xc62-1/Xc62-1-1-421-6-eng.pdf.

15. Toronto overdose action plan: prevention and response. Toronto Public Health; 2017. Retrieved from http://app.toronto.ca/tmmis/ viewAgendaltemHistory.do?item=2017.HL18.3.

16. Recommendations of the mayors' task force on the opioid crisis. Big-City Mayors' Caucus, Federation of Canadian Municipalities; 2017. Retrieved from https://fcm.ca/Documents/issues/Opioid_Crisis_EN.pdf.

17. Factsheet on drug checking in Europe. Trans European Drugs Information (TEDI) Workgroup; 2011. Retrieved from https://www. ecstasydata.org/text/2011/2011_tedi_factsheet_on_drug_checking_in_ europe.pdf.

18. Benschop A, Rabes M, Korf D. Pill testing, ecstasy \& prevention. Eine wis. 2002.

19. Brunt TM, Nagy C, Bücheli A, Martins D, Ugarte M, Beduwe C, et al. Drug testing in Europe: monitoring results of the Trans European Drug Information (TEDI) project. Drug Testing and Analysis. 2017;9(2):188-98.

20. Martins D, Barratt MJ, Pires CV, Carvalho H, Vilamala MV, Espinosa IF et al. The detection and prevention of unintentional consumption of DOx and 25x-NBOMe at Portugal's Boom Festival. Human Psychopharmacology: Clinical and Experimental. 2017;32(3).

21. Hondebrink L, Nugteren-van Lonkhuyzen JJ, Van Der Gouwe D, Brunt TM. Monitoring new psychoactive substances (NPS) in The Netherlands: data from the drug market and the Poisons Information Centre. Drug Alcohol Depend. 2015;147:109-15.

22. Brunt TM, Niesink RJ. The Drug Information and Monitoring System (DIMS) in the Netherlands: implementation, results, and international comparison. Drug Testing and Analysis. 2011;3(9):621-34.

23. Vogels N, Brunt TM, Rigter S, Van Dijk P, Vervaeke H, Niesink RJ. Content of ecstasy in the Netherlands: 1993-2008. Addiction. 2009;104(12):2057-66.

24. Spruit IP. Monitoring synthetic drug markets, trends, and public health. Substance Use and Misuse. 2001;36(1-2):23-47.

25. Giné CV, Vilamala MV, Espinosa IF, Lladanosa CG, Álvarez NC, Fruitós AF, et al. Crystals and tablets in the Spanish ecstasy market 2000-2014: are they the same or different in terms of purity and adulteration? Forensic Science International. 2016;263:164-8.

26. Caudevilla-Gálligo F, Ventura M, Indave Ruiz BI, Fornís I. Presence and composition of cathinone derivatives in drug samples taken from a drug test service in Spain (2010-2012). Human Psychopharmacology: Clinical and Experimental. 2013;28(4):341-4.

27. New federal initiatives to address the opioid crisis. Health Canada; 2017. Retrieved from https://www.canada.ca/en/health-canada/news/2017/11/ new_federal_initiativestoaddresstheopioidcrisis.html.

28. Reducing regulatory barriers to accessing treatment, and new funding for innovative projects. Health Canada; 2018. Retrieved from https://www. canada.ca/en/health-canada/news/2018/03/reducing-regulatory-barriers-toaccessing-treatment-and-new-funding-for-innovative-projects.html.

29. Harper L, Powell J, Pijl EM. An overview of forensic drug testing methods and their suitability for harm reduction point-of-care services. Harm Reduct J. 2017;14(1):52.

30. Maghsoudi N. Comparing models of drug checking services in Canada. Centre on Drug Policy Evaluation; 2018.

31. Barratt MJ, Kowalski M, Maier LJ, Ritter A. Global review of drug checking services operating in 2017. Sydney, Australia: National Drug and Alcohol Research Centre, UNSW Sydney; 2018.

32. Evidence brief: drug checking services as a harm reduction intervention. Public Health Ontario; 2017. Retrieved from https://www. publichealthontario.ca/en/eRepository/Evidence_Brief_Drug_ Checking_2017.pdf.

33. Rhodes T. The 'risk environment': a framework for understanding and reducing drug-related harm. Int J Drug Policy. 2002;13(2):85.

34. Firestone M, Goldman B, Fischer B. Fentanyl use among street drug users in Toronto, Canada: behavioural dynamics and public health implications. Int J Drug Policy. 2009;20(1):90-2.

35. Bayoumi A, Strike C, Brandeau ML, Degani N, Fischer B, Glazier R, et al. Report of the Toronto and Ottawa Supervised Consumption Assessment Study. Toronto: St. Michael's Hospital and the Dalla Lana School of Public Health, University of Toronto; 2012.

36. Rhodes T. Risk environments and drug harms: a social science for harm reduction approach. Int J Drug Policy. 2009;20(3):193-201.
37. Frajzyngier V, Neaigus A, Gyarmathy VA, Miller M, Friedman SR. Gender differences in injection risk behaviors at the first injection episode. Drug Alcohol Depend. 2007;89(2-3):145-52.

38. Evans JL, Hahn JA, Page-Shafer K, Lum PJ, Stein ES, Davidson PJ, et al. Gender differences in sexual and injection risk behavior among active young injection drug users in San Francisco (the UFO Study). J Urban Health. 2003;80(1):137-46.

39. Schiffer K. 'Nothing about us, without us': participation and involvement of drug users in health services and policy making. Amsterdam: Correlation Network; 2011.

40. Magura S. Validating self-reports of illegal drug use to evaluate National Drug Control Policy: a reanalysis and critique. Eval Program Plann. 2010; 33(3):234-7.

41. Lloyd-Smith E, Wood E, Zhang R, Tyndall MW, Montaner JS, Kerr T. Risk factors for developing a cutaneous injection-related infection among injection drug users: a cohort study. BMC Public Health. 2008;8(1):405.

42. Baral S, Holland CE, Shannon K, Logie C, Semugoma P, Sithole B, et al. Enhancing benefits or increasing harms: community responses for HIV among men who have sex with men, transgender women, female sex workers, and people who inject drugs. JAIDS. 2014;66:S319-S28.

43. Werb D, Garfein R, Kerr T, Davidson P, Roux P, Jauffret-Roustide M, et al. A socio-structural approach to preventing injection drug use initiation: rationale for the PRIMER study. Harm Reduct J. 2016;13:25.

44. Doherty MC, Edgar Monterroso MD MPH. Gender differences in the initiation of injection drug use among young adults. J Urban Health. 2000; 77(3):396-414.

45. Tsai JS, Lin GL. Drug-testing technologies and applications. Drugs of Abuse: Springer; 2005. p. 29-69.

46. Day J, Slawson M, Lugo R, Wilkins D. Analysis of fentanyl and norfentanyl in human plasma by liquid chromatography-tandem mass spectrometry using electrospray ionization. Journal of Analytical Toxicology. 2003;27(7):513-6.

47. Proctor J, Larsen K. 'An exceptionally high toll': Fentanyl, overdoses claim 488 in B.C. CBCca. 2016 September 21.

48. Gomes T, Mamdani MM, Paterson JM, Dhalla IA, Juurlink DN. Trends in highdose opioid prescribing in Canada. Can Fam Physician. 2014;60(9):826-32.

49. TPH. Toronto Overdose Information system: calls to paramedic services for suspected opioid overdoses - geographic information. Toronto: Toronto Public Health; 2019.

50. Braun V, Clarke V. Using thematic analysis in psychology. Qualitative Reseach in Psychology. 2006;3(2):77-101.

51. Morse JM. The significance of saturation. Qualitative Health Research 1995. Contract No.: 2.

52. Leung L. Validity, reliability, and generalizability in qualitative research. J Family Med Prim Care. 2015;4(3):324-7.

53. Barbour RS. Checklists for improving rigour in qualitative research: a case of the tail wagging the dog? BMJ (Clinical Research Edition). 2001; 322(7294):1115-7.

54. Grundy C, Steinbach R, Edwards P, Green J, Armstrong B, Wilkinson P. Effect of $20 \mathrm{mph}$ traffic speed zones on road injuries in London, 19862006: Controlled interrupted time series analysis. BMJ. 2009, 339:b4469.

55. Werb D, Strathdee SA, Vera A, Arredondo J, Beletsky L, Gonzalez-Zuniga P, et al. Spatial patterns of arrests, police assault and addiction treatment center locations in Tijuana, Mexico. Addiction. 2016;111(7):1246-56.

56. Marshall BDL, Milloy MJ, Wood E, Montaner JSG, Kerr T. Reduction in overdose mortality after the opening of North America's first medically supervised safer injecting facility: a retrospective population-based study. Lancet. 2011;377(9775):1429-37.

57. Fotheringham AS, Brunsdon C, Charlton M. Geographically weighted regression: the analysis of spatially varying relationships: John Wiley \& Sons; 2003.

58. Teddlie C, Tashakkori A. Foundations of mixed-methods research: integrating quantitative and qualitative approaches in the social and behavioral sciences. USA: Sage Publications Inc; 2009.

59. McNeil R, Small W, Wood E, Kerr T. Hospitals as a 'risk environment': an ethno-epidemiological study of voluntary and involuntary discharge from hospital against medical advice among people who inject drugs. Soc Sci Med. 2014;105:59-66.

60. Agar M. Toward a qualitative epidemiology. Qual Health Res. 2003;13(7): 974-86.

61. Lopez AM, Bourgois P, Wenger LD, Lonvick J, Martinez AN, Kral AH. Interdisciplinary mixed methods research with structurally vulnerable populations: case studies of injection drug users in San Francisco. Int J Drug Policy. 2013;24(2):101-9. 
62. Tupper KW, McCrae K, Garber I, Lysyshyn M, Wood E. Initial results of a drug checking pilot program to detect fentanyl adulteration in a Canadian setting. Drug Alcohol Depend. 2018;190:242-5.

63. U.S. drug overdose deaths continue to rise; increase fueled by synthetic opioids [press release]. Centers for Disease Control and Prevention, 2018-0329 T03:14:28Z 2018. Retrieved from https://www.cdc.gov/media/releases/201 8/p0329-drug-overdose-deaths.html.

64. Deaths related to drug poisoning in England and Wales: 2016 registrations. Office for National Statistics; 2017. Retrieved from https://www.ons.gov.uk/ peoplepopulationandcommunity/birthsdeathsandmarriages/deaths/ bulletins/deathsrelatedtodrugpoisoninginenglandandwales/2016 registrations.

65. Charlois T, editor Safer Nightlife in Europe. 5th Meeting EXASS Network 2009: Council of Europe's Pompidou Group.

66. Hungerbuehler I, Buecheli A, Schaub M. Drug Checking: A prevention measure for a heterogeneous group with high consumption frequency and polydrug use-evaluation of Zurich's drug checking services. Harm Reduc J. 2011;8(1):16

\section{Publisher's Note}

Springer Nature remains neutral with regard to jurisdictional claims in published maps and institutional affiliations.

Ready to submit your research? Choose BMC and benefit from:

- fast, convenient online submission

- thorough peer review by experienced researchers in your field

- rapid publication on acceptance

- support for research data, including large and complex data types

- gold Open Access which fosters wider collaboration and increased citations

- maximum visibility for your research: over $100 \mathrm{M}$ website views per year

At BMC, research is always in progress.

Learn more biomedcentral.com/submissions 